\title{
The coefficient variation of home blood pressure is a novel factor associated with macroalbuminuria in type 2 diabetes mellitus
}

\author{
Emi Ushigome ${ }^{1}$, Michiaki Fukui ${ }^{1}$, Masahide Hamaguchi ${ }^{2}$, Takafumi Senmaru ${ }^{1}$, Kazumi Sakabe ${ }^{1}$, \\ Muhei Tanaka ${ }^{1}$, Masahiro Yamazaki ${ }^{1}$, Goji Hasegawa ${ }^{1}$ and Naoto Nakamura ${ }^{1}$
}

The purpose of this study was to investigate the association between day-by-day variability in home blood pressure (HBP) on 14 consecutive days and macroalbuminuria in patients with type 2 diabetes. We compared the coefficient of variation (CV) of HBP in 858 Japanese patients with and without macroalbuminuria. Next, we analyzed the relationship between the logarithm of urinary albumin excretion (UAE) and the CV of HBP using linear regression analysis. Then, we evaluated the association between the CV of HBP and macroalbuminuria, defined as UAE $\geqslant 300 \mathrm{mg} \mathrm{g}^{-1}$ creatinine, using logistic regression analysis. The CVs of morning and evening systolic blood pressure (SBP) were significantly greater in patients with macroalbuminuria than in those without (8.08 \pm 3.35 vs. $7.19 \pm 2.25 \%, P<0.05$ and $9.01 \pm 3.58$ vs. $7.98 \pm 2.57 \%, P<0.05$, respectively). Multivariate linear regression analyses indicated that the CVs of morning SBP $(P<0.05)$ and diastolic blood pressure (DBP; $P<0.05)$, and those of evening SBP $(P<0.05)$ were the independent explanatory variables for the logarithm of UAE. Multivariate logistic regression analyses also demonstrated that the odds ratio for the CVs of morning SBP, morning DBP and evening SBP for macroalbuminuria were $1.35(P<0.05), 1.29(P<0.05)$ and $1.44(P<0.05)$, respectively. We conclude that the CV of HBP is correlated with macroalbuminuria, independent of the known risk factors, in Japanese patients with type 2 diabetes. Hypertension Research (2011) 34, 1271-1275; doi:10.1038/hr.2011.128; published online 4 August 2011

Keywords: diabetic nephropathy; home blood pressure; variability in blood pressure

\section{INTRODUCTION}

The purpose of long-term care for diabetic patients is to prevent the development of diabetic complications. It is important to control blood pressure (BP) as well as blood glucose to prevent microvascular and macrovascular complications. Home BP (HBP) measurement in the morning has been found to have a strong relationship with target organ damage in several population-based studies and prospective clinical studies. ${ }^{1,2}$ The Ohasama study was a well-known large-scale longitudinal observational study of ambulatory BP monitoring and HBP measurement in a Japanese population. ${ }^{1}$ This study demonstrated that masked hypertension, defined as normal clinic BP and elevated HBP, was closely related to chronic kidney disease, and HBP measurement could be a useful screening strategy to detect chronic kidney disease in the general population. Therefore, the utility of HBP measurement has been widely accepted and self-measurement of HBP is widely used in clinical practice for patients at high risk for chronic kidney disease and patients with cardiovascular disease. Recent studies have explored the association between variability in BP and cardiovascular events, and they demonstrated that variability in BP predicted the risk of stroke and left ventricular hypertrophy. ${ }^{3-5}$ To our knowledge, no reports have explained the relationship between variability in HBP and target organ damage in diabetic patients. Therefore, we investigated the relationship between variability in HBP and diabetic nephropathy in Japanese patients with type 2 diabetes.

\section{METHODS}

\section{Patients}

HBP measurements were performed in patients with type 2 diabetes who regularly attended the diabetes outpatient clinic at Hospital of Kyoto Prefectual University of Medicine and four other general hospitals. We sequentially recruited adult patients, ages 23-89, who visited the facilities between March-October 2008 and March-October 2009. We excluded the winter season in order to avoid variations in BP caused by cold temperatures. We did not institute a BP level criterion for study inclusion. According to the criteria in the Japanese Society of Hypertension Guidelines 2009, ${ }^{6}$ hypertension was defined as a HBP that is $\geqslant 135 / 85 \mathrm{~mm} \mathrm{Hg}$ in untreated patients or any level if the patient was currently being treated for hypertension.

A total of 954 type 2 diabetic patients agreed to participate in this study. First, 31 patients who did not adequately measure HBP were excluded. We also

${ }^{1}$ Department of Endocrinology and Metabolism, Kyoto Prefectural University of Medicine, Graduate School of Medical Science, Kyoto, Japan and ${ }^{2}$ Laboratory of Experimental Immunology, WPI Immunology Frontier Research Center at Osaka University, Osaka, Japan

Correspondence: Dr M Fukui, Department of Endocrinology and Metabolism, Kyoto Prefectural University of Medicine, Graduate School of Medical Science, 465 Kajii-cho, Kawaramachi-Hirokoji, Kamigyo-ku, Kyoto 602-8566, Japan.

E-mail: sayarinapm@hotmail.com

Received 10 February 2011; revised 18 May 2011; accepted 30 May 2011; published online 4 August 2011 
excluded 55 patients for whom urinary albumin excretion (UAE) was not measured. Second, 10 patients who had advanced renal dysfunction (serum creatinine $\geqslant 2.0 \mathrm{mg} \mathrm{dl}^{-1}$ ) were excluded from the analyses. Finally, 858 patients comprised the study population (464 male, 394 female). The diagnosis of type 2 diabetes mellitus was based on the American Diabetes Association criteria. ${ }^{7}$ All patients gave their informed consent to participate in this study.

\section{Study design}

We designed a cross-sectional multicenter study in general hospitals that were located in the Kansai area in Japan. The centers included Hospital of Kyoto Prefectual University of Medicine and the other four general hospitals. These five hospitals had specialized diabetes outpatient clinics, $>500$ beds for hospitalization and examined about 2000 outpatients per day. First, we investigated the relationship between the logarithm of UAE and the coefficient of variation $(\mathrm{CV})$ of $\mathrm{BP}$, which we used as an index of variability in $\mathrm{HBP}$, using a linear regression analysis. Second, we evaluated independent determinants of the logarithm of UAE by multivariate linear regression analysis after adjustment for the following variables and confounding factors, which were known risk factors for nephropathy: sex, age, body mass index, low-density lipoprotein cholesterol, hemoglobin $\mathrm{A}_{1 \mathrm{C}}$, BP level, use of antihypertensive medication and duration of diabetes. Furthermore, we evaluated the association between $\mathrm{CV}$ of $\mathrm{BP}$ with macroalbuminuria, which was defined as UAE $\geqslant 300 \mathrm{mgg}^{-1} \mathrm{Cr}$, by logistic regression models. All procedures were carried out in accordance with institutional and international ethical guidelines for human studies.

\section{Data collection}

Blood samples for biochemical measurements were taken at the hospital. Hemoglobin $A_{1 C}$, serum lipid profile (low-density lipoprotein cholesterol, triglycerides and high-density lipoprotein cholesterol) and other biochemical data were determined by standard laboratory measurements. UAE was measured with an immunoturbidimetric assay. A mean value for UAE was determined from triplicate urine collections. Information including age, duration of diabetes, microvascular complications, macrovascular complications, smoking, alcohol consumption, hypoglycemic medication and antihypertensive medication were obtained at the time of the BP measurement. Alcohol consumption (every day, social or never) and smoking status (current, past, or never) were assessed by interview.

HBP measurements were performed using an automatic device, HEM70801C (Omron Healthcare Co. Ltd, Kyoto, Japan), which uses the cuff oscillometric method to generate a digital display of heart rate and systolic/ diastolic blood pressure (SBP/DBP) values. HEM-70801C uses the identical components and BP determining algorithm to those of another device, HEM705IT, which was previously validated and satisfied the criteria of the British Hypertension Society protocol. ${ }^{8}$

\section{Coefficient of variation}

Patients were instructed to perform triplicate morning and evening BP measurements for 14 consecutive days. Measurements of morning BP were made within $1 \mathrm{~h}$ of waking, before breakfast or taking any drugs, with the patient seated and rested for at least 5 min. ${ }^{9}$ Measurements of evening BP were obtained in a homologous way just before going to bed. The cuff was placed directly around their non-dominant arm and the position of cuff was maintained at the level of the heart. We calculated the standard deviation (s.d.) of HBP for all 14 days, for the first half (measured during days 1-7) and for the latter half (measured during days 8-14) in the morning and in the evening. As an indicator for variability in $\mathrm{BP}$, we defined the $\mathrm{CV}$ of $\mathrm{HBP}$ as s.d. divided by average HBP. Then, we calculated the CV of HBP for all 14 days, the first half and the latter half in the morning and in the evening.

\section{Sample size}

We could not set the sample size before the study because previous reports were not available regarding the relationship between CV of HBP and macroalbuminuria.

\section{Statistical analysis}

Values were expressed as mean \pm s.d. for continuous variables and as a number for categorical variables. UAE showed a skewed distribution, so logarithmic transformation was carried out before performing statistical analyses. Univariate and multivariate linear regression analyses were used to compare the relationship between the logarithm of UAE and risk factors. Univariate and multivariate logistic analyses were used to determine the contribution of the variables to macroalbuminuria. To adjust for the effects of various factors on the logarithm of UAE or the existence of macroalbuminuria, the following factors, which were known risk factors for nephropathy, were considered as covariates: sex, age, body mass index, low-density lipoprotein cholesterol, hemoglobin $\mathrm{A}_{1 \mathrm{C}}$, $\mathrm{BP}$ level, use of antihypertensive medication and duration of diabetes. ${ }^{10,11}$ Odds ratios and $95 \%$ confidence intervals were calculated. Values of $P<0.05$ were considered to be statistically significant. All statistical analyses were performed using SPSS version 11.0J (SPSS Inc. Chicago, IL, USA).

\section{RESULTS}

Clinical characteristics of patients are shown in Table 1 . Of the 858 patients, 73 (8.5\%, 41 male, 32 female) had macroalbuminuria, and $649(75.6 \%)$ had a diagnosis of hypertension. The mean age and use of antihypertensive medications were significantly higher in patients with macroalbuminuria. Morning and evening SBP were significantly higher in patients with macroalbuminuria. The CV of morning and evening SBP in patients with macroalbuminuria were also significantly greater than those without macroalbuminuria.

From the results of univariate linear regression analyses (Table 2), the logarithm of UAE showed a significant positive relationship with: CV of morning SBP and DBP for all 14 days, for the first half and for the latter half; with CV of evening SBP for all 14 days, for the first half and for the latter half; and with CV of evening DBP for all 14 days. Multivariate linear regression analyses indicated that the independent explanatory variable concerning CV of HBP for the logarithm of UAE were as follows; CV of morning and evening SBP for all 14 days and for the latter half, and that of morning DBP for all 14 days, for the first half and for the latter half (Table 2).

Univariate logistic regression analyses demonstrated that $\mathrm{CV}$ of morning SBP and DBP for all 14 days and for the latter half and CV of evening SBP for all 14 days and for the latter half were positively associated with macroalbuminuria (Table 3). Multivariate logistic regression analyses also revealed that $\mathrm{CV}$ of morning SBP and DBP for all 14 days and for the latter half and CV of evening SBP for all 14 days and for the latter half had independent positive associations with macroalbuminuria.

\section{DISCUSSION}

\section{Principal findings}

Herein, we describe a large scale cross-sectional study in Japanese type 2 diabetic patients, which showed that CV of HBP was significantly associated with macroalbuminuria independent of the following known risk factors: sex, age, body mass index, low-density lipoprotein cholesterol, hemoglobin $\mathrm{A}_{1 \mathrm{C}}$, BP level, use of antihypertensive medication and duration of diabetes. As far as we know, this is the first study to indicate a positive association between CV of HBP with macroalbuminuria, independent of the known risk factors.

\section{Interpretations}

Our data show that variability in HBP is associated with macroalbuminuria. This novel finding highlights the importance of HBP measurement. In the future, we would like to investigate the optimal range of $\mathrm{CV}$ of $\mathrm{HBP}$, how to use this knowledge, how to reduce variability and whether such a reduction will improve prognosis. 
Table 1 Clinical characteristics of patients

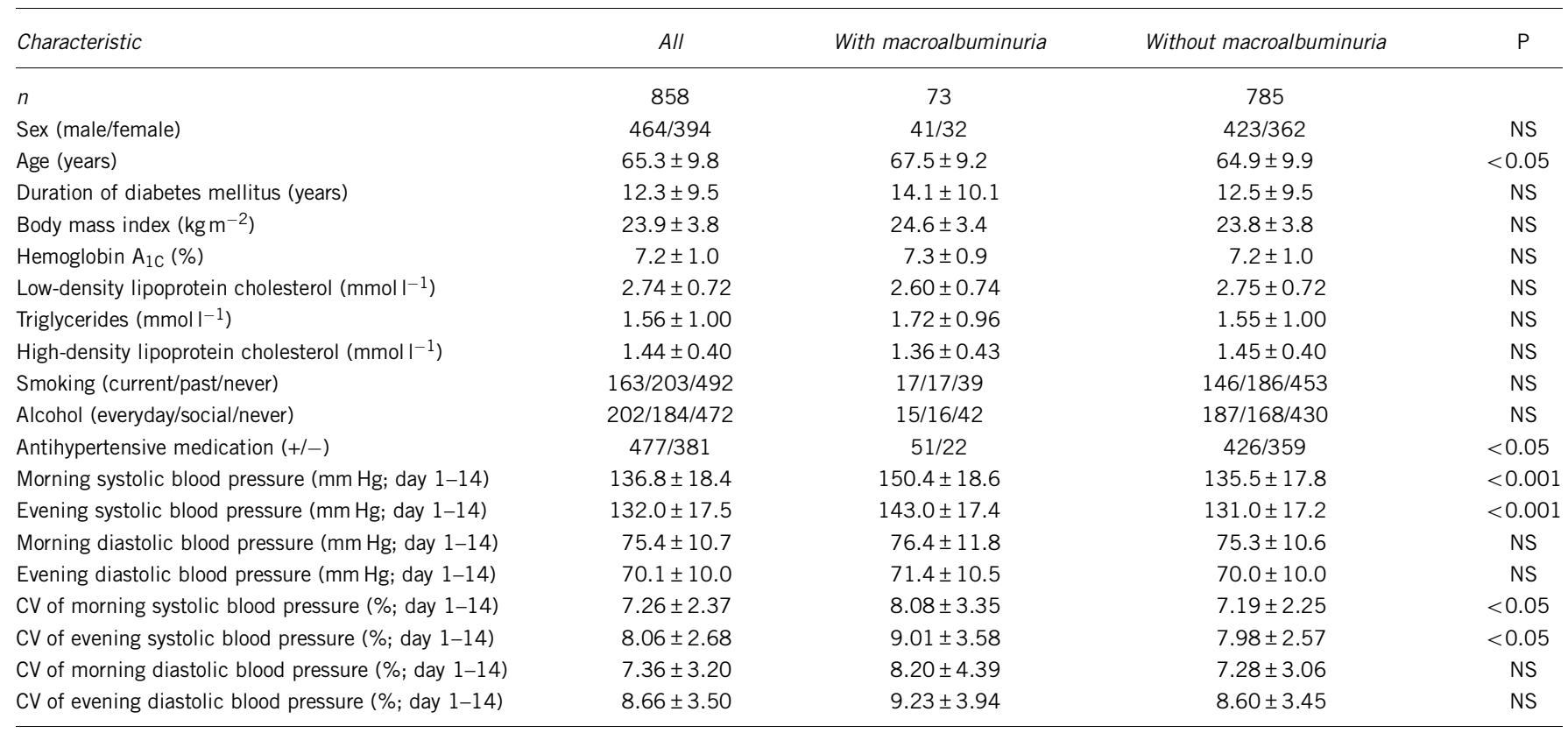

Abbreviations: $\mathrm{CV}$, coefficient of variation; NS, not significant.

Data are means \pm s.d. or number. Mean values were compared using unpaired Student's $t$ test. A $\chi$-test was used to compare categorical variables between the two groups with and without macroalbuminuria.

Table 2 The linear relationship between logarithm of urinary albumin excretion and the CV of blood pressure in Japanese type 2 diabetic patients

\begin{tabular}{|c|c|c|c|c|}
\hline & \multicolumn{2}{|c|}{ Univariate } & \multicolumn{2}{|c|}{ Multivariate adjusted ${ }^{\circledR}$} \\
\hline & $\gamma$ & $P$ & $\beta$ & $P$ \\
\hline $\mathrm{CV}$ of morning SBP (day 1-14) & 0.114 & $<0.05$ & 0.106 & $<0.05$ \\
\hline CV of morning SBP (day 1-7) & 0.067 & $<0.05$ & 0.059 & NS \\
\hline CV of morning SBP (day 8-14) & 0.081 & $<0.05$ & 0.103 & $<0.05$ \\
\hline CV of morning DBP (day 1-14) & 0.13 & $<0.001$ & 0.115 & $<0.05$ \\
\hline CV of morning DBP (day $1-7$ ) & 0.097 & $<0.05$ & 0.083 & $<0.05$ \\
\hline CV of morning DBP (day 8-14) & 0.123 & $<0.001$ & 0.129 & $<0.05$ \\
\hline CV of evening SBP (day 1-14) & 0.118 & $<0.05$ & 0.089 & $<0.05$ \\
\hline CV of evening SBP (day $1-7$ ) & 0.072 & $<0.05$ & 0.064 & NS \\
\hline CV of evening SBP (day 8-14) & 0.094 & $<0.05$ & 0.085 & $<0.05$ \\
\hline CV of evening DBP (day $1-14$ ) & 0.078 & $<0.05$ & 0.057 & NS \\
\hline CV of evening DBP (day $1-7$ ) & 0.055 & NS & 0.047 & NS \\
\hline CV of evening DBP (day 8-14) & 0.057 & NS & 0.044 & NS \\
\hline
\end{tabular}

Abbreviations: CV, coefficient of variation; DBP, diastolic blood pressure; NS, not significant; SBP, systolic blood pressure.

aAdjusted for age, sex, blood pressure level, body mass index, low-density lipoprotein

cholesterol, hemoglobin $A_{1 c}$, duration of diabetes mellitus, use of antihypertensive medication. $\beta$ indicates multiple linear regression coefficient.

Linear regression analyses were used to evaluate a relationship between logarithm of urinary albumin excretion and the $\mathrm{CV}$ of home blood pressure.

Johansson et al. ${ }^{12}$ tried to assess the optimal scheme for HBP measurement in relation to target organ damage and ambulatory BP. The correlations between HBP measurements for 7 days with target organ damage and ambulatory BP increased with the cumulative number of measurement days, although no major increases occurred after day 4 . Therefore, they recommended that HBP should be measured on at least 4 days. This is why we compared the association of macroalbuminuria with CV of HBP for all 14 days, for the first half and for the latter half.
In previous studies, the s.d. or CV of BP was used as an index of variability in HBP. ${ }^{3,13,14}$ The Ohasama study reported that the s.d. of $\mathrm{BP}$ was significantly and positively correlated with the ambulatory BP, but the CV of SBP was independent of SBP. ${ }^{14}$ Mancia et al. ${ }^{15}$ also reported that s.d., but not $\mathrm{CV}$, of $\mathrm{BP}$ was greater in hypertensive subjects than in age-matched normotensive subjects. From these results, we thought that the $\mathrm{CV}$ of $\mathrm{BP}$ was less strongly correlated with BP than s.d. of BP, so we used the CV of HBP as an index of variability in HBP.

Self-measurement of BP and ambulatory BP monitoring are widely used for assessing the actual state of BP. These two methods of BP measurement provide different information. Self-measurement of BP can provide long-term information on variability in BP and average BP measured under relatively stable conditions. In contrast, ambulatory $\mathrm{BP}$ can provide 1-day information on variability in $\mathrm{BP}$ and average BP during daytime, nighttime and $24 \mathrm{~h}$ measured under daily activity. We assessed variability in BP not by ambulatory BP monitoring but by self-measurement of BP. Self-measurement of $\mathrm{BP}$ is more convenient and is more widely used in clinical practice than ambulatory BP monitoring. Moreover, it is able to assess variability in BP over longer periods under relatively controlled conditions.

Previous studies showed that differences in mean HBP and morning and evening HBP are determinants of UAE. ${ }^{16}$ In our study, we also examined relationships between morning SBP, evening SBP or the difference between morning and evening SBP, and UAE or the existence of macroalbuminuria. The multivariate linear regression analyses showed that morning and evening SBP were significantly and positively related with the logarithm of UAE $(P<0.001)$. Multivariate logistic regression analyses also revealed that morning and evening SBP had independent positive associations with macroalbuminuria $(P<0.001)$. Furthermore, the $\mathrm{CV}$ of $\mathrm{BP}$ was independently positively associated with the logarithm of UAE and macroalbuminuria, even after adjustment for BP level. 
Table 3 Univariate and multivariate adjusted odds ratios of the $\mathrm{CV}$ of blood pressure for macroalbuminuria in Japanese type 2 diabetic patients

\begin{tabular}{|c|c|c|c|c|}
\hline & \multicolumn{2}{|c|}{ Univariate } & \multicolumn{2}{|c|}{ Multivariate adjusted ${ }^{a}$} \\
\hline & Odds ratio $(95 \% \mathrm{Cl})$ & $P$ & Odds ratio $(95 \% \mathrm{Cl})$ & $P$ \\
\hline CV of morning SBP (day $1-7$ ) & $1.22(0.969-1.524)$ & NS & $1.23(0.929-1.621)$ & NS \\
\hline CV of morning SBP (day 8-14) & $1.31(1.049-1.644)$ & $<0.05$ & $1.51(1.144-1.982)$ & $<0.05$ \\
\hline CV of morning DBP (day 1-14) & $1.24(1.032-1.483)$ & $<0.05$ & $1.29(1.251-1.332)$ & $<0.05$ \\
\hline CV of evening SBP (day 1-14) & $1.45(1.144-1.838)$ & $<0.05$ & $1.44(1.095-1.890)$ & $<0.05$ \\
\hline CV of evening SBP (day $1-7$ ) & $1.11(0.881-1.394)$ & NS & $1.11(0.853-1.435)$ & NS \\
\hline $\mathrm{CV}$ of evening SBP (day 8-14) & $1.52(1.207-1.903)$ & $<0.001$ & $1.54(1.176-2.029)$ & $<0.05$ \\
\hline CV of evening DBP (day $1-14$ ) & $1.15(0.954-1.382)$ & NS & $1.14(0.926-1.405)$ & NS \\
\hline CV of evening DBP (day $1-7$ ) & $1.12(0.886-1.418)$ & NS & $1.12(0.868-1.457)$ & NS \\
\hline CV of evening DBP (day 8-14) & $1.15(0.912-1.448)$ & NS & $1.15(0.887-1.496)$ & NS \\
\hline
\end{tabular}

Abbreviations: $\mathrm{Cl}$, confidence interval; $\mathrm{CV}$, coefficient of variation; DBP, diastolic blood pressure; NS, not significant; SBP, systolic blood pressure.

Data are expressed as odds ratio $(95 \% \mathrm{Cl})$.

${ }^{a}$ Adjusted for age, sex, body mass index, blood pressure level, low-density lipoprotein cholesterol, hemoglobin $A_{1 C}$, duration of diabetes mellitus, use of antihypertensive medication.

Logistic analyses were used to determine the contribution of the $\mathrm{CV}$ of home blood pressure to the existence of macroalbuminuria.

The causal relationship between variability in HBP and macroalbuminuria could not be determined clearly because this study had a cross-sectional design. However, animal studies suggest several possibilities. First, it has been reported that in sinoaortic-denervated rats (an experimental model of high variability in BP without hypertension), variability in BP is a more critical determinant than BP level for cardiac damage, renal lesions and aortic hypertrophy. ${ }^{17}$ Second, Eto et al. ${ }^{18}$ demonstrated that sinoaortic denervation reduced endothelium-dependent vasorelaxation and enhanced neointimal formation after balloon injury. These results suggest that increased BP fluctuations, independent of average BP level, may contribute to atherogenesis. Miao et al. ${ }^{19}$ proposed the hypothesis that hemodynamic effects and activation of the renin-angiotensin system contribute to organ damage induced by increased variability in BP in sinoaortic-denervated rats. Higher variability in BP may cause greater variation in tissue perfusion. Cellular metabolism may be disturbed by these variations. Furthermore, large variations in BP may cause a direct lesion in arterial endothelial cells. It is well known that angiotensin II can cause vasoconstriction, cell growth and proliferation, cardiovascular hypertrophy, angiogenesis and augmentation of sympathetic activity. Most of these actions of angiotensin II may contribute to organ damage. One study found that plasma renin activity was increased in the acute phase of sinoaortic denervation. ${ }^{20}$ Furthermore, previous human studies have also shown increases in ambulatory $\mathrm{BP}$ variability to be related to atherosclerosis, urinary protein excretion and cardiovascular disease in Japanese patients with type 2 diabetes. ${ }^{21-23}$ It was postulated that the presence of advanced atherosclerosis and impaired baroreflex sensitivity, which was associated with diabetic neuropathy, could lead to increased variability in BP in diabetic patients.

\section{CONCLUSIONS}

This study revealed that CV of HBP correlated with macroalbuminuria, independent of the known risk factors, in Japanese patients with type 2 diabetes. Large prospective studies are needed to confirm CV of $\mathrm{HBP}$ as a risk factor for macroalbuminuria in patients with type 2 diabetes.

\section{CONFLICT OF INTEREST}

The authors declare no conflict of interest.

\section{ACKNOWLEDGEMENTS}

We thank Dr Shinobu Inada in Kyoto Prefectural University of Medicine, Dr Atsushi Omoto, Dr Toru Tanaka and Dr Wataru Fukuda in Kyoto First Red Cross Hospital, Dr Haruhiko Atsuta in Kyoto Second Red Cross Hospital, Dr Masayoshi Ohnishi, Dr Shin-ichi Mogami and Dr Yoshihiro Kitagawa in Osaka General Hospital of West Japan Railway Company and Dr Yohei Oda in Social insurance Kyoto Hospital, for their assistance with researching data; Naoko Higo, Machiko Hasegawa and Terumi Kaneko in Kyoto Prefectural University of Medicine, for teaching patients how to measure their blood pressure; and Sayoko Horibe, Hiroko Kawamura and Sanae Utena in Kyoto Prefectural University of Medicine for their secretarial assistance.

1 Ohkubo T, Imai Y, Tsuji I, Nagai K, Kato J, Kikuchi N, Nishiyama A, Aihara A, Sekino M, Kikuya M, Ito S, Satoh H, Hisamichi S. Home blood pressure measurement has a stronger predictive power for mortality than does screening blood pressure measurement: a population-based observation in Ohasama, Japan. J Hypertens 1998; 16: 971-975.

2 Sega R, Trocino G, Lanzarotti A, Carugo S, Cesana G, Schiavina R, Valagussa F, Bombelli M, Giannattasio C, Zanchetti A, Mancia G. Alterations of cardiac structure in patients with isolated office, ambulatory, or home hypertension: data from the general population (Pressione Arteriose Monitorate E Loro Associazioni [PAMELA] Study). Circulation 2001; 104: 1385-1392.

3 Mancia G, Bombelli M, Facchetti R, Madotto F, Corrao G, Trevano FQ, Grassi G, Sega R. Long-term prognostic value of blood pressure variability in the general population: results of the Pressioni Arteriose Monitorate e Loro Associazioni Study. Hypertension 2007; 49: 1265-1270.

4 Ekbom T, Dahlöf B, Hansson L, Lindholm LH, Odén A, Scherstén B, Wester PO. The stroke preventive effect in elderly hypertensives cannot fully be explained by the reduction in office blood pressure-insights from the Swedish Trial in Old Patients with Hypertension (STOP-Hypertension). Blood Press 1992; 1: 168-172.

5 Frattola A, Parati G, Cuspidi C, Albini F, Mancia G. Prognostic value of 24-h blood pressure variability. J Hypertens 1993; 11: 1133-1137.

6 Ogihara T, Kikuchi K, Matsuoka H, Fujita T, Higaki J, Horiuchi M, Imai Y, Imaizumi T, Ito S, Iwao H, Kario K, Kawano Y, Kim-Mitsuyama S, Kimura G, Matsubara H, Matsuura H, Naruse M, Saito I, Shimada K, Shimamoto K, Suzuki H, Takishita S, Tanahashi N, Tsuchihashi T, Uchiyama M, Ueda S, Ueshima H, Umemura S, Ishimitsu T, Rakugi H, Japanese Society of Hypertension Committee. The Japanese Society of Hypertension Guidelines for the Management of Hypertension (JSH 2009). Hypertens Res 2009; 32: 3-107.

7 The Expert Committee on the Diagnosis and Classification of Diabetes Mellitus. Report of the expert committee on the diagnosis and classification of diabetes mellitus. Diabetes Care 2002; 25: S5-S20.

8 Coleman A, Freeman P, Steel S, Shennan A. Validation of the Omron 705IT (HEM-759E) oscillometric blood pressure monitoring device according to the British Hypertension Society protocol. Blood Pressure Monit 2006; 11: 27-32.

9 Imai Y, Otsuka K, Kawano Y, Shimada K, Hayashi H, Tochikubo O, Miyakawa M, Fukiyama K. Japanese Society of Hypertension (JSH) guidelines for self-monitoring of blood pressure at home. Hypertens Res 2003; 26: 771-782. 
10 Kamoi K, Miyakoshi M, Soda S, Kaneko S, Nakagawa O. Usefulness of home blood pressure measurement in the morning in type 2 diabetic patients. Diabetes Care 2002; 25: 2218-2223.

11 UK Prospective Diabetes Study (UKPDS) Group. Intensive blood-glucose control with sulphonylureas or insulin compared with conventional treatment and risk of complications in patients with type 2 diabetes (UKPDS 33). Lancet 1998; 352: 837-853.

12 Johansson JK, Niiranen TJ, Puukka PJ, Jula AM. Optimal schedule for home blood pressure monitoring based on a clinical approach. J Hypertens 2010; 28: 259-264.

13 Kato T, Kikuya M, Ohkubo T, Satoh M, Hara A, Obara T, Metoki H, Asayama K, Hirose T, Inoue R, Kanno A, Totsune K, Hoshi H, Satoh H, Imai Y. Factors associated with day-byday variability of self-measured blood pressure at home: the Ohasama study. Am J Hypertens 2010; 23: 980-986.

14 Imai Y, Aihara A, Ohkubo T, Nagai K, Tsuji I, Minami N, Satoh H, Hisamichi S. Factors that affect blood pressure variability. A community-based study in Ohasama, Japan. Am J Hypertens 1997; 10: 1281-1289.

15 Mancia G, Ferrari A, Gregorini L, Parati G, Pomidossi G, Bertinieri G, Grassi G, di Rienzo M, Pedotti A, Zanchetti A. Blood pressure and heart rate variabilities in normotensive and hypertensive human beings. Circ Res 1983; 53: 96-104.

16 Kario K, Hoshide S, Shimizu M, Yano Y, Eguchi K, Ishikawa J, Ishikawa S, Shimada K. Effect of dosing time of angiotensin II receptor blockade titrated by self-measured blood pressure recordings on cardiorenal protection in hypertensives: the Japan Morning Surge-Target Organ Protection (J-TOP) study. J Hypertens 2010; 28: 1574-1583.
17 Miao $\mathrm{CY}$, Xie HH, Zhan LS, Su DF. Blood pressure variability is more important than blood pressure level in determination of end-organ damage in rats. J Hypertens 2006; 24: 1125-1135.

18 Eto M, Toba K, Akishita M, Kozaki K, Watanabe T, Kim S, Hashimoto M, Sudoh N, Yoshizumi M, Ouchi Y. Reduced endothelial vasomotor function and enhanced neointimal formation after vascular injury in a rat model of blood pressure lability. Hypertens Res 2003; 26: 991-998.

19 Miao CY, Tao X, Gong K, Zhang SH, Chu ZX, Su DF. Arterial remodeling in chronic sinoaortic-denervated rats. J Cardiovasc Pharmacol 2001; 37: 6-15.

20 Padilha JU, Krieger EM. Alteration in baroreceptor function in rats produces typical pressure changes during sleep. J Cardiovasc Pharmacol 1987; 10: S194-S198.

21 Tamura K, Tsurumi Y, Sakai M, Tanaka Y, Okano Y, Yamauchi J, Ishigami T, Kihara M, Hirawa N, Toya Y, Yabana M, Tokita Y, Ohnishi T, Umemura S. A possible relationship of nocturnal blood pressure variability with coronary artery disease in diabetic nephropathy. Clin Exp Hypertens 2007; 29: 31-42.

22 Masuda S, Tamura K, Wakui H, Kanaoka T, Ohsawa M, Maeda A, Dejima T, Yanagi M, Azuma K, Umemura S. Effects of angiotensin II type 1 receptor blocker on ambulatory blood pressure variability in hypertensive patients with overt diabetic nephropathy. Hypertens Res 2009; 32: 950-955.

23 Eguchi K, Ishikawa J, Hoshide S, Pickering TG, Schwartz JE, Shimada K, Kario K. Night time blood pressure variability is a strong predictor for cardiovascular events in patients with type 2 diabetes. Am J Hypertens 2009; 22: 46-51. 